\title{
Proposal of Automatic Dust Catcher Robot of Clothes Using Kinect
}

\author{
Natsuki Nakamoto ${ }^{\mathrm{a}}$, Yuhki Kitazono ${ }^{\mathrm{a}^{*}}$ \\ ${ }^{a}$ National Institute of Technology, Kitakyushu College,5-20-1 Shii, Kokuraminami-ku, Kitakyushu-city, Fukuoka 802- \\ 0985, Japan \\ *Corresponding Author: kitazono@kct.ac.jp
}

\begin{abstract}
If you come into contact with others with dust or animal hair on your clothes, there is a high possibility that you will make the other person develop an allergy or not be able to establish good communication. Thus, it is necessary to remove dusts and more from your clothes before going out. However, this work is done manually, which is timeconsuming and time-consuming. To solve this problem, last time we developed a robot that automatically removes dust from clothes on hangers with a brush. The robot holds and pinches the clothes between two brushes and removes the dust by moving the brushes from top to bottom. It also removes dust from the entire clothes by repeatedly extending the arm with the brush attached and removing the dust. However, this robot could only operate for one garment at a time. In this paper, we develop a robot that can continuously care for multiple clothes. First, this robot rotates the hanger rack. Next, use the Kinect camera to look at the front of the brush and stop the rotation of the hanger rack when the clothes come. Third, remove the dust from the clothes in the same way as the previous robot we created. By repeating the above operation, multiple clothes can be removed dust in succession. The operation time of this robot is about two minutes. All the user has to do is put the clothes on the hanger, press the start button on the robot, and the robot will remove the dust from the clothes. While the robot is running, the user can spend his time doing other things. For example, by using this robot, you can make better use of your valuable morning time for yourself instead of using it to dust your clothes.
\end{abstract}

Keywords: clothes, dust, brush.

\section{Introduction}

House dust, mites, and animals can cause allergies ${ }^{(1)-(4)}$. It is dangerous to come in contact with allergy sufferers with these substances on your clothes ${ }^{(5)-(7)}$. Therefore, it is necessary to remove dust and other particles from clothes when there is a possibility of contacting with people.

Also, appearance is a quite important point in giving a positive impression on others ${ }^{(8)}$. According to the questionnaire on marriage activities conducted in 2020 by Partner Agent, a dating agency operated by Tameny Corporation says $48.4 \%$ of men and $65.0 \%$ women of 978 men and women aged 30-39 who have done marriage activities ranked appearance and cleanliness as the most important factor in determining the impression of a partner or the factor that makes a good impression in marriage activities $^{(9)}$. This is the most common answer for both men and women. In addition, according to the questionnaire conducted in 2019 by this corporation shows, $54.7 \%$ of 320 men and women aged 20-39 answered that cleanliness is an important factor in what they look for in a marriage partner $^{(10)}$.

Thus, wearing clean clothes is one of the things we need to do to maintain good health for ourselves and others, and to build good relationships with others. In order to wear clean clothes, it is necessary to wash, iron out wrinkles, and remove dust from clothes. In this paper, we focused on the task of removing dust from clothes.

Since the average member of household in Japan is two people, we consider the amount of dust generated at this time ${ }^{(11)}$. According to "On the generation of dust in rooms", a collection of reports by the Architectural Institute of Japan in 1966, the dust generation rate when two people are in one room is $2.44 \times 10-3(\mathrm{~m} 3 / \mathrm{s})^{(12)}$.

Many people have pets. According to the national survey of the Pet Food Association's in 2020, 6.8 million households have 8.49 million dogs and 5.51 million households have 9.64 million cats ${ }^{(13)}$. Dogs and cats shed a 


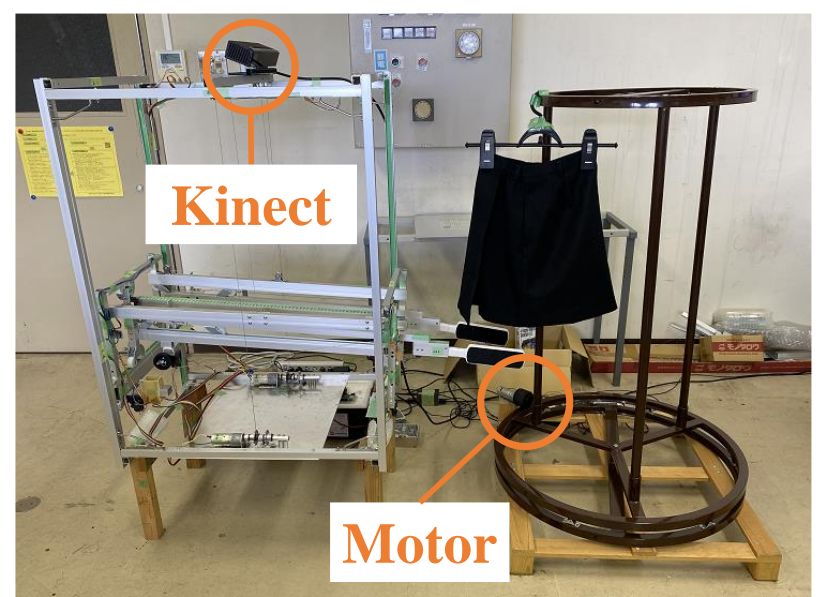

Fig. 1. Robot.

lot of hair. One Labrador retriever sheds 238 hairs per 30 minutes, which is 11424 hairs a day ${ }^{(14)}$. Many pet owners are troubled by their pets' hair loss ${ }^{(15)(16)}$ Kärcher Japan Co. surveyed 500 men and women who own a dog or cat in $2019^{(17)}$. According to it, the biggest concern for the majority of them when cleaning was dirt around the floor caused by pet hair. Animal hair, which falls on the floor in large quantities, adheres to our clothes as we sit or walk. In this way, if you keep animals such as dogs and cats indoors, a lot of animal hair will stick to your clothes in addition to dust.

Dust and animal hair on clothes are mostly removed by using rolls of adhesive tape, special brushes, and other useful items. However, these need to be done manually, which can be a big burden if you have a lot of clothes or if you have a dog or cat.

Many people spend a lot of time grooming themselves in the morning before going to work ${ }^{(18)}$. And many people feel that they don't have time in the morning. According to the survey on how people spend their mornings on workdays conducted in 2012 by Lion Corporation shows, $65.1 \%$ of 1000 office worker aged 20-50 answered that don't have enough time on the morning of the day that have to go to work $^{(19)}$. In addition, in this survey, when asked how much they could afford to pay for a minute of their busy morning, the average answer was 531 yen.

In the past, we have developed robots that control brushes to remove dust from clothes ${ }^{(20)}$. However, this robot could only dust one piece of garment at a time. In this paper, we propose a robot that can operate continuously on multiple clothes by combining a robot developed in the past with a rotating hanger $\operatorname{rack}^{(21)}$. This robot first rotates the hanger rack when the start button is pressed. Next, using the Kinect camera, when a garment comes in front of the brush, it stops

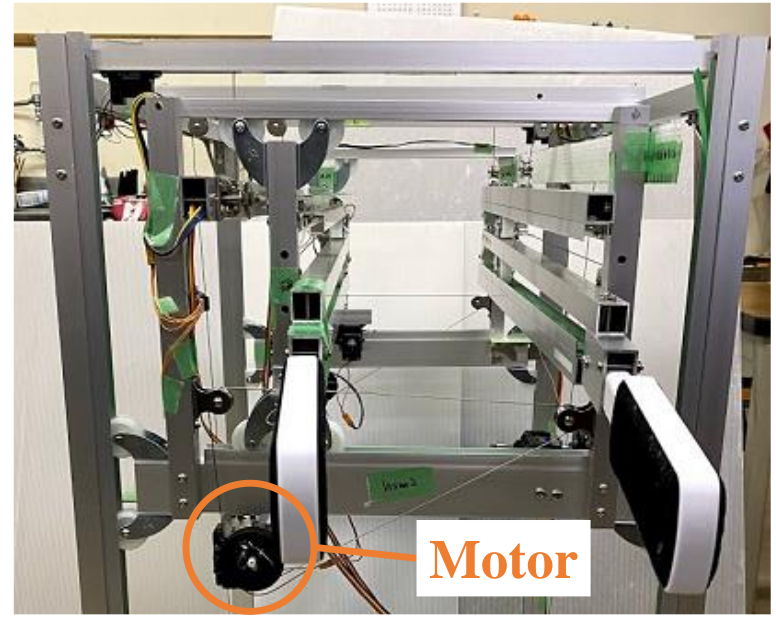

(a) Drive unit 1 .

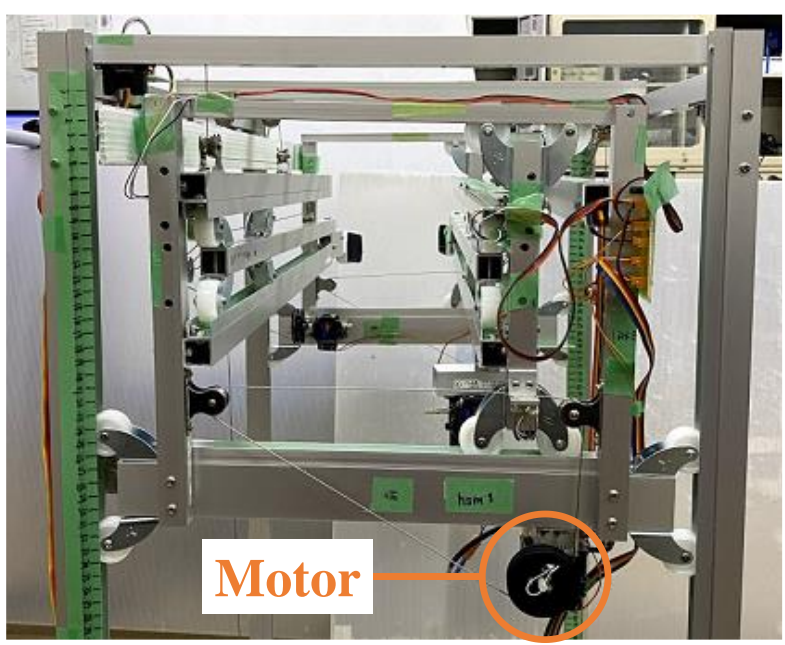

(b) Drive unit 2.

Fig. 2. Drive units.

rotating the hanger rack and removes the dust from the clothes in the same way as the previous robot we developed. By repeating the above process, multiple clothes can be dusted in succession.

\section{Automatic Dust Catcher Robot of Clothes Using Kinect}

The overall view of the prototype machine is shown in Figure 1. The rotating hanger rack on the right is where you hang your clothes. The size of the robot's main body on the left is about $740 \mathrm{~W} \times 495 \mathrm{D} \times 1360 \mathrm{H}(\mathrm{mm})$, and the arm with the brush (Kyowa Kogyo Co., Ltd. MUGENBRUSH) juts out 380 830 (mm). The robot's main body is something we have developed in the past, and it does the job of removing dust from clothes. The robot's main body holds the clothes between two brushes and moves them from top to bottom to remove the dust. The robot then extends the 


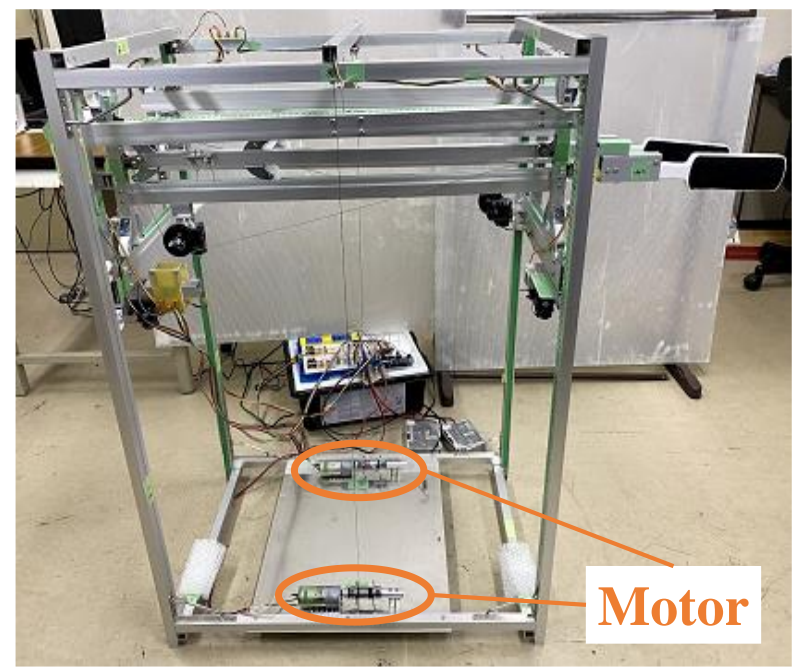

Fig. 3. Moves up and down.

brushes several times and repeats the above operation to remove dust from the entire clothes. The above motions are controlled as the axes that hold, move up and down, and extend the clothes. However, the main body of the robot alone could only remove dust from one piece of garment at a time. In this paper, we propose a robot that can operate continuously to multiple clothes by combining the robot body with a rotating hanger rack. The robot rotates a hanger rack and uses Kinect (Microsoft, Xbox One) to perform image processing to continuously remove dust from multiple clothes. The hanger rack is rotated by a motor (TAMIYA, $540 \mathrm{~K} 300$ ) and controlled by a motor driver (Pololu, MD03A). The mechanism of each axis of the robot body is shown in Sections 2.1 2.3, and the control method of the hanger rack by image processing is shown in Section 2.4.

\subsection{Axis to hold clothes}

A photo of the pinching axis of the prototype machine is shown in Figure 2. As shown in Fig. 2, only one of the brushes has a drive unit on each side. The two drive units are called the drive unit 1 in Fig. 2(a) and the drive unit 2 in Fig. 2(b), respectively. The two drive units are driven simultaneously to pinch the clothes. The brushes are driven up to $160 \mathrm{~mm}$ in the pinching direction. This motion is achieved by winding the wire using a motor (TAMIYA, 4Speed Worm Gearbox H.E.) and pulleys. The motor is run using a 5(V) DC power supply, and the drive direction is controlled using a motor driver (Toshiba, TA7291P). In addition, a distance sensor (Sharp, GP2Y0E03) is installed in each of the two driving sections, and the width of the sandwich is controlled using an Arduino.

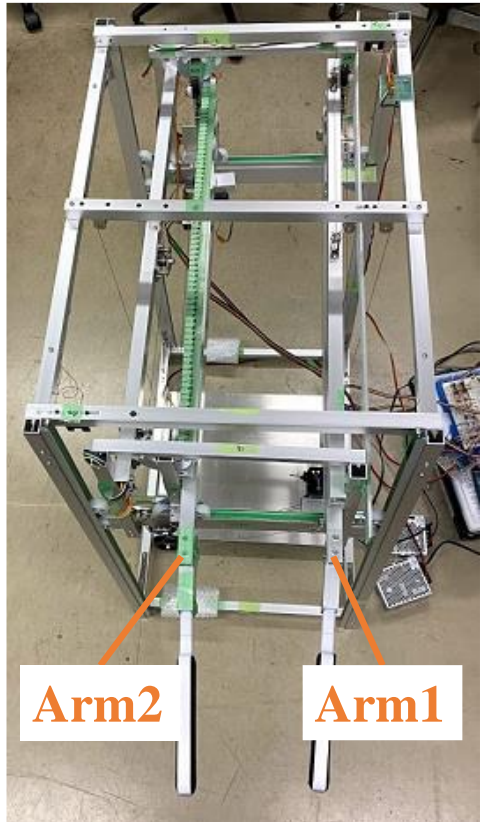

Fig. 4. Arms.

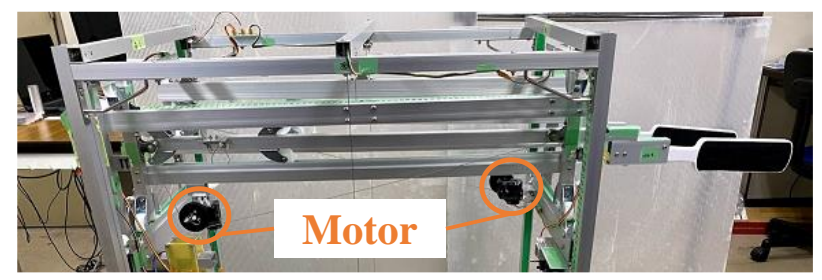

Fig. 5. Extend.

\subsection{Axis to move the brush up and down}

Fig. 3 shows the mechanism for moving the brush up and down in the prototype. The two brushes are moved up and down by simultaneously driving the two mechanism for extending brushes as shown Fig. 3. Fig. 4 shows the top view of the arms with the brushes attached. The arms are called Arm 1 and Arm 2 respectively, as shown in Fig. 4. The brushes are driven up and down the maximum of $520(\mathrm{~mm})$. The motor (TAMIYA, AO-8037 Geared Motor 540K300) and the wires are used for this movement. The motor runs on a $12(\mathrm{~V})$ DC power supply and winds the wire with a pipe. The motor driver (Pololu, VNH3SP30) is used to control the drive direction. In addition, a distance sensor (Sharp, GP2Y0A02) is installed in each of the two drive sections, and the height at which it is moved can be controlled using an Arduino.

\subsection{Axis to extend the brush}

Fig. 5 shows the mechanism for extending the brushes of the prototype as in Section 2.2, we distinguish the arms to 


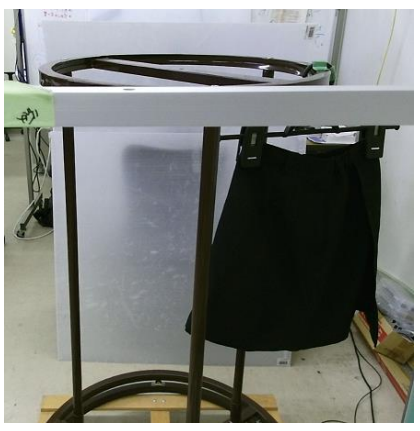

(a) Not in front.

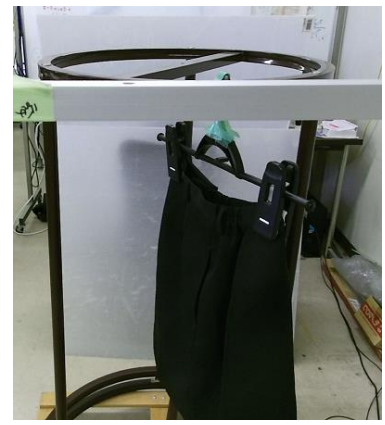

(b) $30 \%$

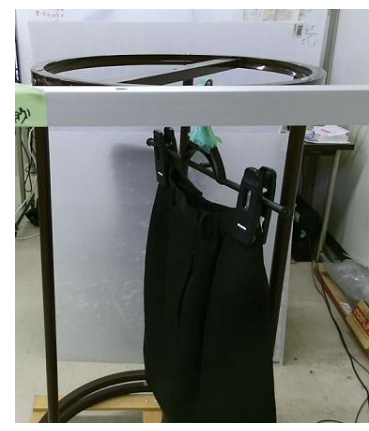

(c) $50 \%$.

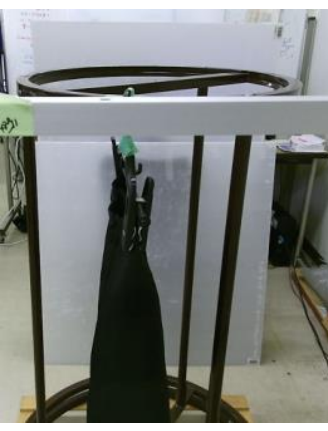

(d) End of rotation.

Fig. 6. Short pants as seen from Kinect.

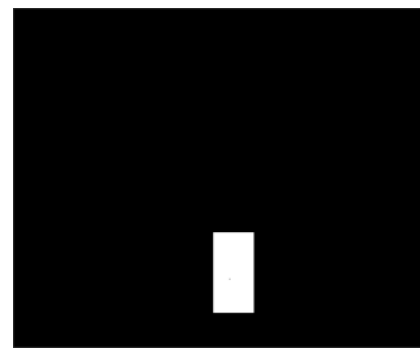

(a) Not in front.

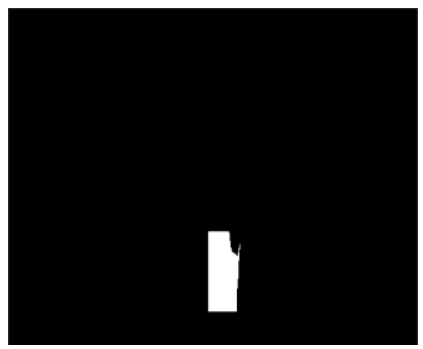

(b) $30 \%$.

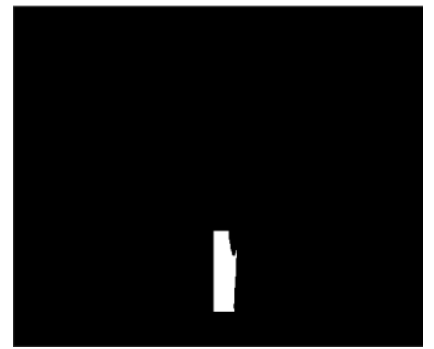

(c) $50 \%$.

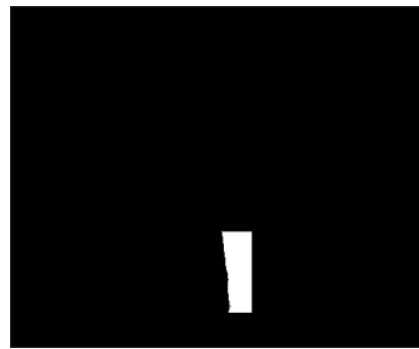

(d) End of rotation.

Fig. 7. Binarized image with narrowed range.

be driven as in Fig. 9. The brushes are driven the maximum of $450(\mathrm{~mm})$. This movement is achieved by using a motor (TAMIYA, 4-Speed Worm Gearbox H.E.) and a pulley to wind the wire. The motor is run using a $5(\mathrm{~V})$ DC power supply, and the drive method is controlled using a motor driver (Toshiba, TA7291P). In addition, the axis to be extended can be seen by the distance sensor (Sharp, GP2Y0A21YK0F), and the distance to extend the brush can be controlled using the Arduino.

\subsection{Controlling a Hanger Rack by Image Processing}

To operate continuously on multiple pieces of garment, image processing is performed using Kinect to control the rotating hanger rack. As shown in Fig. 1, a Kinect is placed on top of the robot body so that the robot can see the clothes hung on the hanger rack. The robot acquires distance images from the Kinect and performs image processing. The hanger rack stops rotating when the image processing confirms that the clothes are in front of the robot body. Next, the robot removes the dust from the clothes. When the robot finishes its work, the hanger rack starts to rotate again. By repeating the above operation, the robot can operate continuously on multiple clothes.

When the clothes are in front of the robot body, the distance from Kinect to the clothes is about $650(\mathrm{~mm})$. When there is an object between $600 \sim 800(\mathrm{~mm})$ from the Kinect, we assumed that the clothes are in front of the robot body. First, the distance image acquired from the Kinect was converted into a binarized image as black when there is an object within the above distance range and white when there is not. At this time, a part of the hanger rack will also be within the above distance. Thus, when the clothes were in front of the robot body, we defined the range in which the clothes were definitely and only visible in the image. This range was defined as within 250 300 horizontal and 280 380 vertical pixels of an image of size $512 \times 424$.The presence or absence of clothes was judged according to how much of the black displayed when an object was in $600 \sim 800$ $(\mathrm{mm})$ was within that range. In this paper, we consider the blurring of the image caused by the rotation of the hanger rack and the error in the control of the motor. Stop the motor when the black area reaches $50 \%$ within the range where the clothes can be seen. Fig. 6 shows the clothes as seen from the Kinect until the hanger rack stops rotating. Fig. 7 shows a binarized image with a narrowed range at the time of Fig. 6 . Fig. 6 and 7 are obtained from the Kinect, so the left and right sides are reversed.

The Kinect is operated by a PC, and the motors that operate the robot body and rotate the rotating hanger rack are controlled by an Arduino. First, the Arduino rotates the rotating hanger rack; the $\mathrm{PC}$ starts Kinect and sends a signal from the $\mathrm{PC}$ to the Arduino when the clothes come to the 
front of the robot body using the above identification method. After confirming the reception with the Arduino, the robot stops the rotation of the hanger rack, and then performs the action of removing the dust from the clothes with the robot body. When the robot is finished, it rotates the hanger rack again and sends a signal from the Arduino to the PC. After confirming the reception by the $\mathrm{PC}$, the Kinect will start discriminating again. By repeating the above actions, the robot can operate for multiple clothes. However, the above operation has a problem that the hanger rack keeps rotating forever. To solve this problem, the robot operation was terminated when the hanger rack made one rotation. First, we measured the time it takes for the hanger rack to make one rotation. On the Arduino side, the time while the hanger rack is rotating is measured and compared with the time for one rotation to control it. Since the time required for the hanger rack used in this paper to make one rotation is about 23 seconds, the robot is controlled to end its operation when the rotation exceeded 26 seconds to account for errors.

\section{Experiment}

In this paper, we control a rotating hanger rack in order to make the robot work continuously on multiple pieces of clothes. A $100 \%$ polyester, small and medium size short pants was used as a sample of the clothes. Using Kinect, we verified the operation of the hanger rack using the discrimination method described in section 2.4. By using the above discrimination method, we verified whether the hanger rack could be controlled so that the clothes always stopped in front of the robot's main body.

The hanger rack was controlled using the method described in section 2.4. We hung two pairs of short pants on the hanger rack and moved the robot until the hanger rack made one rotation and automatically finished. As a substitute for the original motion of the robot's main body, we simulated the motion of the entire robot by blinking the LED five times every 0.5 seconds. The hanger rack was rotated 10 times using the method described above, and the $\mathrm{x}$ coordinate of the center of gravity of the short pants in the image was measured and observed to see if it always stopped in front of the robot body. The x-coordinate of the center of gravity of the clothes was measured by performing the binarization process of the distance image described in section 2.4 on the entire distance image acquired, while inverting it in black and white. When the garment was in front of the robot body, the x-coordinate of the center of gravity the short pants in the image was 300 , so this was used as the reference. The difference between the measured $\mathrm{x}$ coordinate of the center of gravity of the short pants and the reference value is shown in Table 1. Table 1 shows that the error is small, so the clothes always stop in front of the robot's main body.

\section{Conclusions}

In this paper, we have proposed and simulated a system that can operate on multiple pieces of dusty clothes. We believe that this robot can solve the problem of removing dust of only one single garment at a time, which has been a problem in previous paper. This robot can remove dust from all the clothes hung on the rotating hanger rack. The user only needs to hang the clothes on the hanger rack and collect the clothes after the robot's operation. Therefore, the time spent on removing dust of clothes can be used for other things. For example, the valuable time in the morning can be effectively used for other things instead of removing dust of clothes.

In the future, it is necessary to verify whether the robot can remove dust of multiple pieces of garment by operating the entire system with the robot itself. Another issue is to improve the stability of the axes of the robot body, which has been an issue in previous papers. In addition, it would be more convenient if the robot could perform removing dust of operations for clothes of different materials and sizes.

\section{References}

(1) Yasunobu Tuduki and Mituo Narita: "Twin cases of anaphylaxis caused by ticks in okonomiyaki flour", Journal of the Japanese Society of Internal Medicine, Vol. 104, No. 5, pp.986-990, 2015

(2) NICHIMO BIOTICS CO., LTD.: "Types of Allergies What are allergies?", http://immubalance.jp/about/allergy-7/, 2021/08/20

(3) Itsuo Suzuki, Fumihisa Okada, Kimiaki Uchimura, Kazuo Ito, Takashi Mitsubayashi, Tooru Akasaka and Kazuichi Maeda: "DIAGNOSIS AND TREATMENT OF CAT ALLERGY AND DOG ALLERGY IN ASTHMATIC CHILDREN", The Japanese Journal of Pediatric Allergy and Clinical Immunology, Vol. 1, No. 1, pp.25-31, 1987

(4) Satoshi Seno, Tadao Enomoto, Yoshihiro Dake, Yuko Saito, Hiroki Ikeda, Hiroko Funakoshi, Hideyo Sogo, Akira Shibano, Takema Sakota, Mikio Suzuki and Yoshiro Yazawa: "Epidemic Study of Pet Allergy in Wakayama Prefecture", Nippon Jibiinkoka Gakkai Kaiho, Vol. 106, No. 7, pp.750-753, 2003

(5) Mitsuhiko Nambu: "Indoor Allergen Control in Allergic Children", Journal of the Japanese Society of Pediatric Allergy, Vol. 24, No. 2, pp.203-216, 2010 
(6) Ryo Muko, Hiroshi Matsuda and Akane Tanaka: "Animal model of skin allergy - Screening and evaluation of functional substances", Functional Food Research, Vol. 14, pp.23-29, 2018

(7) Akira Akasawa: "Can an Allergic Child Have a Pet? From a Pro's Perspective", Journal of the Japanese Society of Pediatric Allergy, Vol. 28, No. 1, pp.58-65, 2014

(8) Keiga Abe: "Literature Guide about Social Attitude in Immersive Virtual Environment", Cognitive Science, Vol. 26, No. 4, pp.509-515

(9) Tameny: "What I changed for marriage activity: Clothes are ranked first for both men and women", Vol. 146, https://www.p-a.jp/research/report 146.html, 2021/08/20

(10) Tameny: "The number one requirement for a marriage partner is someone with a sense of cleanliness.", Vol. 144, https://www.p-a.jp/research/report 144.html, 2021/08/20

(11) Statistics Bureau, Ministry of Finance: "Prefecture, Number of general households by number of persons in household and number of persons in household by type of household", https://www.stat.go.jp/data/nihon/02.html, 2021/08/20

(12) Fujii Syouichi, Konno Keiichi, Kimura Kikuji and Yoshizawa Susumu: "3013 The amount of dust generated indoors", Proceedings of the Architectural Institute of Japan, Extra Issue, Extra Edition, Academic Abstracts, Vol. 41, p. 395-, 1966

(13) Pet Food Association of Japan: "National Survey of Dog and Cat Ownership - Key Indicators and Summary -", https://petfood.or.jp/data/chart2020/3.pdf, 2021/08/20

(14) Yamada Masaru: "Hair Loss in Labrador Retrievers", Japan Assistance Dog Science Research, Vol. 2, No. 1, pp.36-40, 2008

(15) Iris Ohyama: "Cat Census in 2019", https://www.irisplaza.co.jp/media/A14025807205, 2021/08/20

(16) au insurance Co.: "Survey on Pet Health", https://www.au-sonpo.co.jp/corporate/news/detail208.html, 2021/08/20

(17) Kärcher Japan Co.: "Survey on Cleaning and Pets", https://prtimes.jp/main/html/rd/p/000000010.00002299 5.html, 2021/08/20

(18) Do House Inc.: "Survey results on morning activities", https://www.dohouse.co.jp/news/research/20150421/, 2021/08/20

(19) Lion Co.: "Survey on how people spend their mornings on workdays", https://prtimes.jp/main/html/rd/p/000000002.00000429 0.html/, 2021/08/22

(20) Natsuki Nakamoto and Yuhki Kitazono: "Proposal of Automatic Dust Catcher Robot of Clothes", Proceedings of the 9th IIAE International Conference on Industrial Application Engineering 2021, pp.243-250, 2021

(21) Ryunosuke Miyamoto and Yuhki Kitazono: "Development of Robot to Fold T-shirt in the State of Hanging", The Japanese Journal of the Institute of
Industrial Applications Engineers, Vol. 3, No. 1, pp.2433, 2015 